dose averaged throughout the whole lung, presumably because the actual sites of critical tissues had not yet been identified. A comprehensive model for deposition and clearance of dust from the airways was now available and could be used to calculate doses in different parts of the respiratory system, for example, to the cells of the bronchial epithelium, which is a possible site for the induction of cancer by radon. It has been suggested that the lymph nodes may not be critical sites because observed malignancies in experimental animals are largely pulmonary. There is a need to identify the radiation sensitive tissues and to examine the ICRP concept of dose commitment which implies that risk is related to the radiation dose integrated over 50 years without allowance for the latent period between induction and manifestation of cancer and for the lifetime of the critical cells.

The practical implications in respect of emergency reference levels of radiation following nuclear emergencies were outlined by Miss P. M. Bryant (National Radiological Protection Board, Harwell). Because of the present greater understanding of both the tissues at risk and the metabolism of radionuclides such as ${ }^{90} \mathrm{Sr}$ there is a possibility that these levels may be increased.

\section{ENTOMOLOGY \\ Emergence Times}

from our Insect Physiology Correspondent

IT has long been known that the eclosion-emergence-of different insects tends to occur at a specific time of the day or night, and that individuals not quite ready to achieve eclosion one day must wait until the same time next day. That was described many years ago by Corbet for emerging dragonflies. In the current lingo an act or process controlled in time by a circadian clock of this kind is termed a "gated event", on the helpful analogy of a gate which is opened only at fixed times of day and for fixed periods. The emergence of Drosophila from the pupa is a good example described by Pittendrigh and Skopik in 1970.

There are several examples of the gating process being centred in the brain. That is so in the photoperiodic termination of pupal diapause in Antheraea (Williams and Adkisson, 1964). And in this same silk moth, Truman and Riddiford (1970) showed that under the action of the appropriate photoperiod certain neurosecretory cells in the brain liberate an "eclosion hormone" which switches the entire behaviour from pupal to adult type. This includes release of the labial gland secretion which dissolves the silk cocoon, and all the other acts involved in escaping from the old cuticle, expansion of the wings and lysis of the intersegmental muscles. Secretion of the eclosion hormone is evoked by the photoperiod to which the pupa is exposed; but liberation of the hormone at a particular time of day is gated by an inherent circadian rhythm in the brain.

In a recent report Truman (J. Exp. Biol., 57, 805 ; 1972) describes a study of the corresponding process of moulting during the larval stages and shows that the timing of the moults is not controlled by a similar mechanism. A direct correlation exists between the time of ecdysis and the time of release of the prothoracotropic hormone (PTTH) from the brain, which does not suggest that ecdysis is controlled by a circadian timing mechanism. The time of ecdysis is sensitive to temperature, which suggests that a developmental process is involved. Moreover the synchrony of ecdysis within a group of larvae is maintained even in larvae ligated at the neck: the head is not needed to trigger ecdysis. In fact, the time of shedding the larval cuticle is determined by a developmental process which requires a fixed number of hours (depending upon temperature) after the gated release of the PTTH from the brain. For this process a longer time is required in Antheraea as the larva increases in size; thus each successive ecdysis occurs at a progressively later time of day.
The nature of the stimuli that induce secretion of the PTTH, which acts on the prothoracic gland to cause the release of the moulting hormone, ecdysone, is not known in the case of caterpillars. But once these stimuli have been received the brain releases hormone from the neurosecretory cells only during a specific time of day-the "gating period". Although the timing and duration of these gating periods, during which hormone is released, are more or less independent of temperature, the percentage of the population which is able to exploit a given gate, being dependent on a developmental process, is markedly influenced by temperature. It is interesting to find these two different mechanisms for the timing of ecdysis operating respectively in the caterpillar and in the chrysalis of the same insect.

\section{PROTEINS \\ Innards in situ}

from our Molecular Biology Correspondent Some interesting new offerings in the current journals concern themselves with aspects of the chemical and conformational constraints that act on a polypeptide segment and its side chains when it forms part of a native globular protein. How, for instance, to assess the effect of a unique environment within a protein on the behaviour of an ionizing group is a problem that has

\title{
Reversion of HGPRT- Cells
}

MUTANT cells resistant to particular drugs, coupled with cell fusion techniques, are being routinely used today by somatic cell geneticists to produce hybrid cells for genetic analysis. In particular, mutant mouse A9 cells and mouse RAG cells which lack hypoxanthine-guanine phosphoribosyl transferase activity (HGPRT ${ }^{-}$) and so are resistant to 8-azaguanine have been widely used in fusion experiments with cells lacking thymidine kinase $\left(\mathrm{TK}^{-}\right.$) activity and are therefore resistant to bromodeoxyuridine. When HGPRTcells are fused to $\mathrm{TK}^{-}$cells the hybrids can be selected by plating in Littlefield's HAT medium in which the parental cells cannot grow but the hybrids survive because of complementation. But, as Shin, Caneva, Schildkraut, Klinger and Siniscalco point out in Nature New Biology next week (February 14), this selection procedure rests on the assumption that the mutant parental cells do not revert, and they find, contrary to expectation, that both A9 and RAG mutants do indeed revert from the HGPRT - phenotype to the HGPRT ${ }^{+}$ phenotype at a low but significant rate.
Because reversion of these mutant A9 and RAG cells had never been reported, Caneva et al. chose them for an attempt to detect transformation of mammalian cells. They exposed A9 and RAG cells to HeLa cell DNA in the presence of inactivated Sendai virus to facilitate uptake of the DNA and they also set up the appropriate control experiments. Much to their surprise mouse cells able to grow in HAT medium and containing HGPRT activity could be selected from both experimental and control experiments.

Moreover, electrophoretic analysis of the HGPRT in these cells revealed that the enzyme was murine, not human. Apparently, therefore, in conditions which promote cell fusion, A9 cells and RAG cells revert at rates of $1.8 \times 10^{-8}$ and $9.0 \times 10^{-8}$ respectively. Why this should be the case when the cells do not revert if plated in HAT medium in the absence of Sendai virus and the nature of the genetic changes in the parental "mutant" cells and the revertants are intriguing problems. Clearly these findings have far reaching implications for somatic cell geneticists who are using these particular cells. 